ARTICLE

https://doi.org/10.1038/s41467-020-15069-3

\title{
Ruthenium anchored on carbon nanotube electrocatalyst for hydrogen production with enhanced Faradaic efficiency
}

Do Hyung Kweon (10) 1, Mahmut Sait Okyay (D) 2, Seok-Jin Kim (10 1, Jong-Pil Jeon', Hyuk-Jun Noh', Noejung Park (i) ${ }^{2}$, Javeed Mahmood (1) ${ }^{1 凶} \&$ Jong-Beom Baek (iD) ${ }^{1 凶}$

Developing efficient and stable electrocatalysts is crucial for the electrochemical production of pure and clean hydrogen. For practical applications, an economical and facile method of producing catalysts for the hydrogen evolution reaction (HER) is essential. Here, we report ruthenium (Ru) nanoparticles uniformly deposited on multi-walled carbon nanotubes (MWCNTs) as an efficient HER catalyst. The catalyst exhibits the small overpotentials of 13 and $17 \mathrm{mV}$ at a current density of $10 \mathrm{~mA} \mathrm{~cm}^{-2}$ in $0.5 \mathrm{M}$ aq. $\mathrm{H}_{2} \mathrm{SO}_{4}$ and $1.0 \mathrm{M}$ aq. $\mathrm{KOH}$, respectively, surpassing the commercial Pt/C (16 mV and $33 \mathrm{mV})$. Moreover, the catalyst has excellent stability in both media, showing almost "zeroloss" during cycling. In a real device, the catalyst produces $15.4 \%$ more hydrogen per power consumed, and shows a higher Faradaic efficiency (92.28\%) than the benchmark Pt/C (85.97\%). Density functional theory calculations suggest that Ru-C bonding is the most plausible active site for the HER.

\footnotetext{
${ }^{1}$ School of Energy and Chemical Engineering / Center for Dimension-Controllable Organic Frameworks Ulsan National Institute of Science and Technology (UNIST), 50 UNIST, Ulsan 44919, South Korea. ${ }^{2}$ School of Natural Science Ulsan National Institute of Science and Technology (UNIST), 50 UNIST, Ulsan

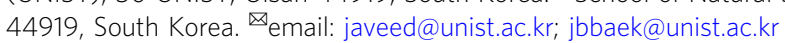


G iven the ongoing depletion of fossil fuels and growing global environmental challenges, the search for carbon less (or free) energy is taking on increasing importance in energy engineering. Among carbon-free energy sources, hydrogen $\left(\mathrm{H}_{2}\right)$, is particularly popular because it contributes no environmental pollutants ${ }^{1}$. The most promising eco-friendly and economical way to produce pure hydrogen is by electrochemical water splitting ${ }^{2-5}$. To ensure the hydrogen evolution reaction (HER) is efficient and continuous, the catalyst must promote proton reduction with minimal overpotential, to minimize additional energy consumption ${ }^{6,7}$. This requirement has made the efficient production of hydrogen using electrochemical catalysts a challenge for scientists over the last several decades ${ }^{8-14}$.

Platinum (Pt) is still considered the benchmark catalyst for the HER, with low overpotentials, small Tafel slopes and high exchange current densities due to its optimum binding force with hydrogen ${ }^{15}$. However, in addition to soaring cost and scarcity, Pt has poor electrochemical stability, which is associated with leaching in corrosive electrolytes and irreversible aggregation of Pt nanoparticles by Ostwald ripening ${ }^{16,17}$, limiting its practical applications. In order to replace Pt, efforts have been devoted to developing earth abundant element-based catalysts for HER, e.g., phosphates $^{18}$, carbides ${ }^{19,20}$, oxides ${ }^{21}$, and transition metal sulfides ${ }^{15,22}$. However, they typically suffer from both limited electrochemical activity and durability.

Recent efforts have focused on designing new catalysts with superior activity and durability compared to commercial $\mathrm{Pt}^{23-25}$. Among the many metal-based catalysts evaluated for HER catalysis, ruthenium $(\mathrm{Ru})$, one of the platinum group metals, has been widely tested, because of its low-cost $(1 / 3 \text { the price of } \mathrm{Pt})^{26}$, high HER efficiency, and stability $25,27,28$. In principle, HER efficiency is closely related to the strength of the metal-hydrogen $(\mathrm{M}-\mathrm{H})$ bonds on the surface of the catalysts ${ }^{29-37}$ and the overpotential required for hydrogen reduction. The Gibbs free energy $\left(\Delta \mathrm{G}_{\mathrm{H}}\right)$ of the $\mathrm{Ru}-\mathrm{H}$ bond is very close to that of the optimum $\mathrm{Pt}-\mathrm{H}$ bond at the center of the volcanic plot for HER $25,38,39$. But even though $\mathrm{Ru}$ has potentially high electrochemical HER activity, it is prone to agglomerate, because it has a much larger cohesive energy than $\mathrm{Pt}^{40}$. To resolve this issue, a strategy of uniformly dispersing and sequestering Ru nanoparticles in a two-dimensional (2D) carbon structure was developed, and it demonstrated excellent HER performance with low overpotentials, outstanding durability and high turnover frequencies in both acidic and alkaline conditions ${ }^{25}$.

Developing methods to produce active but low-cost catalysts remains one of the most crucial obstacles to the realization of a hydrogen economy. Among various approaches, carbon-based materials have attracted interest as low-cost supports for active HER catalysts. Various advanced electrocatalysts have been fabricated by incorporating electrochemically active transition metals into one- or two-dimensional carbon nanostructures, including carbon nanotubes ${ }^{41}$ and graphene nanosheets ${ }^{42}$. These conductive supports are important because they enable the mass production of highly efficient and stable catalysts at low-cost. And in addition to the activity of the catalytic metal nanoparticles, the conductive supports can also make a significant contribution to the overall catalytic performance. For efficient catalysis, the catalytic nanoparticles need to be dispersed and stabilized on an appropriate substrate.

Here, we demonstrate that an electrocatalyst of $\mathrm{Ru}$ nanoparticles anchored on multiwalled carbon nanotube (Ru@MWCNT) is capable of catalysing HER with excellent activity and stability. The Ru@MWCNT catalyst exhibits superior HER activity to Ru@MWCNT and commercial Pt/C catalysts in both acidic and alkaline media. Notably, Ru carboxylate complex is formed through the introduction of carboxylic acid groups (-COOH) on MWCNT to form uniform and small Ru nanoparticles. This suggests the formation of $\mathrm{Ru}$ nanoparticles, $\mathrm{Ru}-\mathrm{C}$ and $\mathrm{Ru}-\mathrm{O}$ bonds through Extended X-ray absorption fine structure (EXAFS). In the actual water-splitting system construction and analysis, Ru@MWCNT produces $15.4 \%$ more hydrogen per power consumption than commercial $\mathrm{Pt} / \mathrm{C}$ and Faradaic efficiency $(92.28 \%)$ is higher than $\mathrm{Pt} / \mathrm{C}(85.97 \%)$. Density functional theory (DFT) calculations identify the $\mathrm{Ru}-\mathrm{C}$ structure as the most plausible active site structure with most stable energies for hydrogen binding energies of possible $\mathrm{H}$ binding sites. The Ru@MWCNT catalysts comprising Ru-C sites as reported herein have appropriate hydrogen binding energies for HER, and strong $\mathrm{Ru}-\mathrm{C}$ bonding energies reflects the excellent stability.

\section{Results}

Preparation and characterization of catalyst. A simple schematic diagram of the $\mathrm{Ru}$ nanoparticle-impregnated MWCNT $(\mathrm{Ru} @ M W C N T)$ catalyst is shown in Fig. 1. Commercial MWCNTs were mildly oxidized with nitric acid to introduce oxygenated functional groups (specifically, carboxylic acids, $-\mathrm{COOH}$ ) on the surface of MWCNT. With abundant carboxylic acids on the surface of the MWCNT, the Ru ions $\left(\mathrm{Ru}^{3+}\right)$ can be easily adsorbed on the surface of the MWCNT, by forming Ru carboxylate complexes ${ }^{43}$. Individual $\mathrm{Ru}^{3+}$ ions were then directly reduced to $\mathrm{Ru}^{0}$ nanoparticles in the presence of sodium borohydride (NaBH4) to form Ru@MWCNT. Subsequent heattreatment (thermal reduction) under inert conditions further reduced the $\mathrm{Ru}$ nanoparticles and oxygenated groups for improved HER performance. Extended X-ray absorption fine structure (EXAFS) spectroscopy was used to analyze the formation of Ru carboxylate complex and local structural environment of Ru@MWCNT catalyst before and after heat-treatment (Supplementary Fig. 1). As a reference, Ru acetylacetonate, containing pristine $\mathrm{Ru}-\mathrm{O}$ bonds was used to confirm $\mathrm{Ru}-\mathrm{O}$ bonding. The Fourier-transformed (FT) $k^{2}$-weighted EXAFS spectrum of the reference $\mathrm{Ru}$ acetylacetonate exhibits the major peak at around $1.5 \AA$ A, corresponding to $\mathrm{Ru}-\mathrm{O}$ coordination. Ru@MWCNT before heat-treatment also has $\mathrm{Ru}-\mathrm{O}$ coordination, which con-

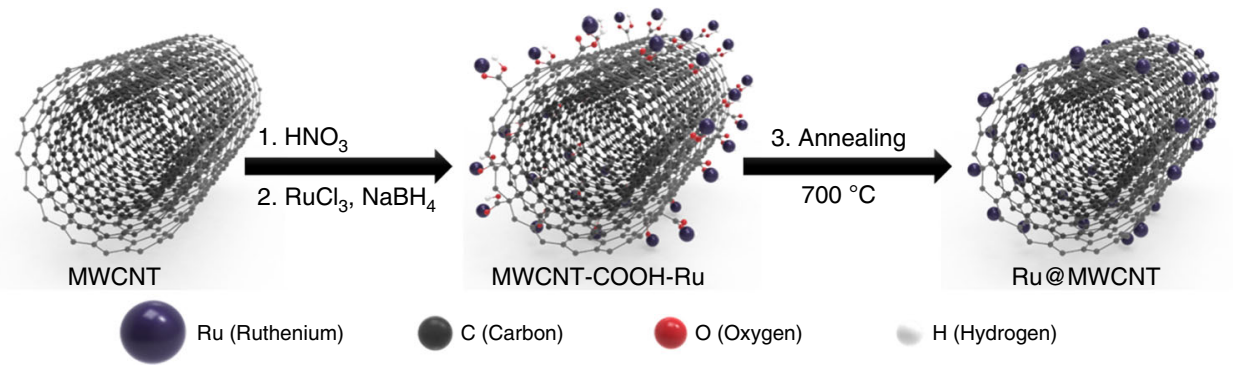

Fig. 1 Schematic illustration of the process steps for forming Ru@MWCNT catalyst. 

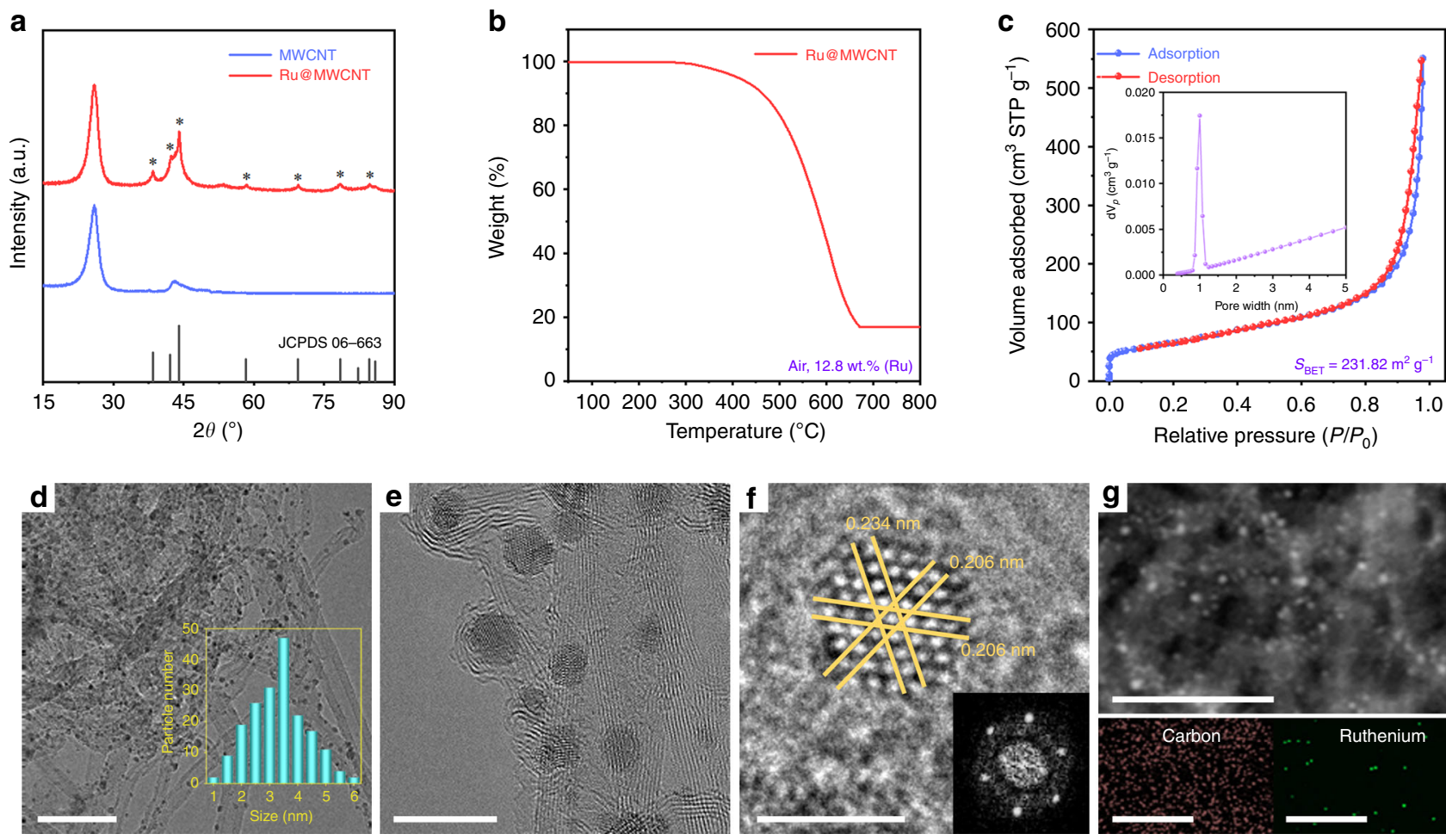

Fig. 2 Structural and morphological characterization of the Ru@MWCNT. a Powder XRD pattern. Asterisks in Ru@MWCNT denote Ru crystal (JCPDS 06-663). b TGA curve under air atmosphere at a ramping rate of $10^{\circ} \mathrm{C} \mathrm{min}-1$. c Nitrogen ( $\mathrm{N}_{2}$ ) adsorption-desorption isotherms at $77 \mathrm{~K}$. The inset in $\mathbf{c}$ shows the pore size distribution. The specific surface area was calculated using the BET method. d, e TEM images. The inset in (d) shows the size distribution of Ru nanoparticles. $\mathbf{f}$ The HR-TEM image is focused on an Ru nanoparticle, showing the high crystallinity of the Ru elements, and their compact packing in the lattice. The inset in $\mathbf{f}$ is the corresponding FFT pattern. $\mathbf{g}$ High-angle annular dark-field scanning transmission electron microscopy (HAADFSTEM) image and scanning transmission electron microscopy coupled energy-dispersive X-ray spectroscopy (STEM-EDS) element mapping of Ru@MWCNT. Scale bar: d 50 nm; e 10 nm; f 2 nm; g 100 nm.

firms the $\mathrm{Ru}$ carboxylate coordination. However, after heattreatment, Ru@MWCNT shows that the peak at $1.5 \AA$ was slightly shifted to $1.6 \AA$, indicating the formation of $\mathrm{Ru}-\mathrm{C}$ coordination 44 . The main peak at $2.4 \AA$ is associated with $\mathrm{Ru}-\mathrm{Ru}$ coordination in $\mathrm{Ru}$ nanoparticles ${ }^{44}$. These results indicate the formation of $\mathrm{Ru}$ carboxylate complexes, which help to form the smaller and more uniform $\mathrm{Ru}$ nanoparticles during the heattreatment. To determine the optimum conditions, the Ru@MWCNT samples were heat-treated at different temperatures. The sample annealed at $700{ }^{\circ} \mathrm{C}$ showed the best HER catalytic performance in both acid and alkaline electrolytes (Supplementary Fig. 2).

The crystal structure of the Ru@MWCNT was analyzed using a high-power X-ray diffraction (HP-XRD) pattern (Fig. 2a). The peak observed at $25.6^{\circ}$ belongs to the (002) plane of the MWCNT. The other peaks at $38.5,42.2,44.1,58.4$ and $69.6^{\circ}$ can be assigned to the (100), (002), (101), (102), and (110) planes of the hexagonal $\mathrm{Ru}$ crystals. The average size of the $\mathrm{Ru}$ nanoparticles on the Ru@MWCNT was calculated to be $3.4 \mathrm{~nm}$ using the Scherrer equation. X-ray photoelectron spectroscopy (XPS) was used to analyze the chemical composition of the Ru@MWCNT (Supplementary Fig. 3). In the high-resolution C 1 s spectrum, the peak at $284.6 \mathrm{eV}$ is associated with the graphitic $\mathrm{C}-\mathrm{C}$ bonds of the MWCNT. The peak at $280.4 \mathrm{eV}$ is related to the atomic state of the $\mathrm{Ru}^{0}$ species in the Ru@MWCNT. The bulk Ru content of Ru@MWCNT was determined by thermogravimetric analysis (TGA) in air, and was $\sim 12.8 \mathrm{wt} \%$ (Fig. 2b). The value is in good accordance with the elemental analysis (Supplementary Table 1). The nitrogen $\left(\mathrm{N}_{2}\right)$ adsorption-desorption isotherm was obtained to calculate the specific surface area $\left(S_{\mathrm{BET}}\right)$ using the Brunauer-
Emmett-Teller (BET) method. The $S_{\mathrm{BET}}$ of the Ru@MWCNT was found to be $231.82 \mathrm{~m}^{2} \mathrm{~g}^{-1}$ (Fig. 2c). Considering the high specific surface area and small Ru nanoparticles, the Ru@MWCNT catalyst was expected to display good HER performance.

The morphology of the Ru@MWCNT was explored by field emission scanning electron microscope (FE-SEM) and transmission electron microscopy and (TEM). The SEM images of the Ru@MWCNT revealed a clean and smooth surface morphology (Supplementary Fig. 4). The TEM images of the Ru@MWCNT clearly confirmed that the $\mathrm{Ru}$ nanoparticles were uniformly anchored to the surface of the MWCNT. The particle size distribution was in the range of $2-5 \mathrm{~nm}$ and the average size was $3.4 \mathrm{~nm}$ (Fig. 2d, e and Supplementary Fig. 5). Due to small particle size, and the uniform and narrow particle size distribution, a large number of $\mathrm{Ru}$ active sites are likely to be exposed, while the MWCNT provides an efficient electron pathway. High-resolution TEM images of the single Ru nanoparticle and the corresponding fast Fourier transform (FFT) pattern showed that the Ru elements were compactly packed into the hexagonal lattice (Fig. 2f) ${ }^{25}$, which precisely agreed with the XRD pattern (Fig. 2a). The uniform distribution of $\mathrm{Ru}$ nanoparticles on the surface of the MWCNT was further confirmed by scanning transmission electron microscopy (STEM) image and corresponding energy-dispersive X-ray spectroscopy (EDS) elemental mapping images (Fig. 2g).

Electrochemical HER activity and stability of Ru@MWCNT catalyst. The Ru@MWCNT catalyst was evaluated for electrochemical HER performance in a $\mathrm{N}_{2}$-saturated $0.5 \mathrm{M}$ aq. $\mathrm{H}_{2} \mathrm{SO}_{4}$ 
a

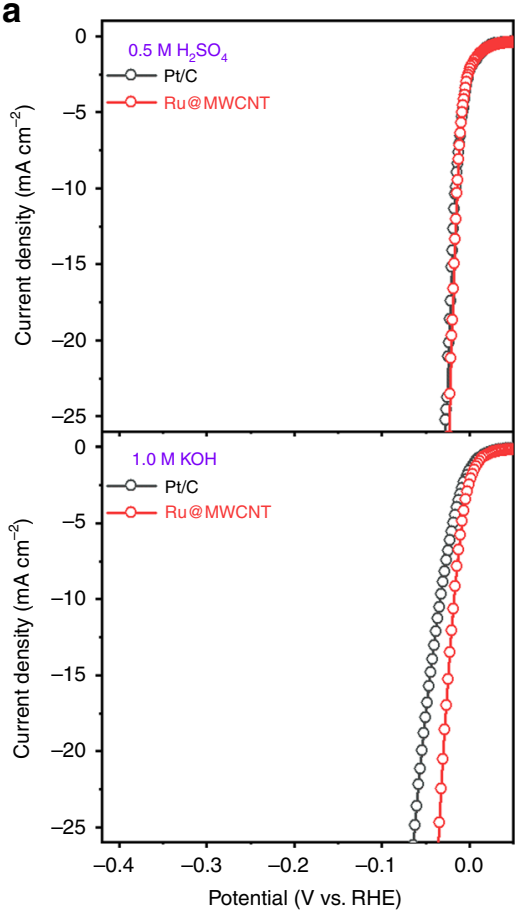

b

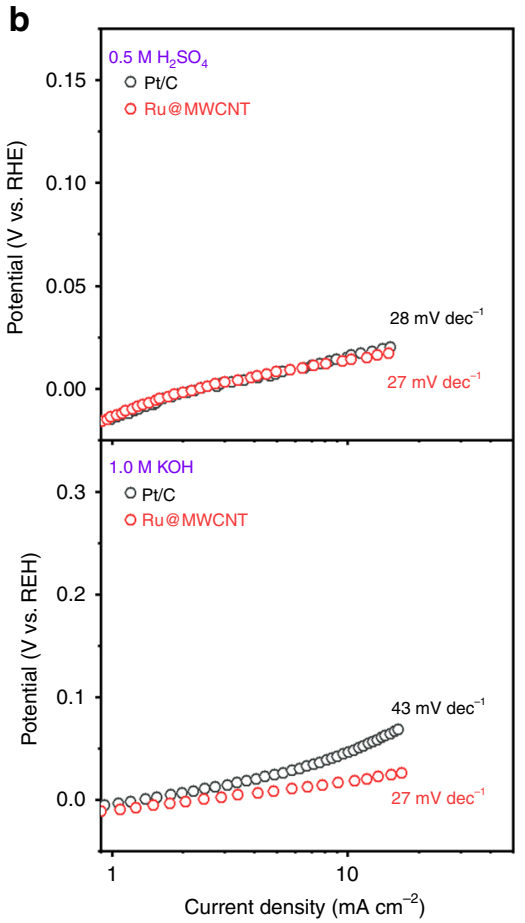

c

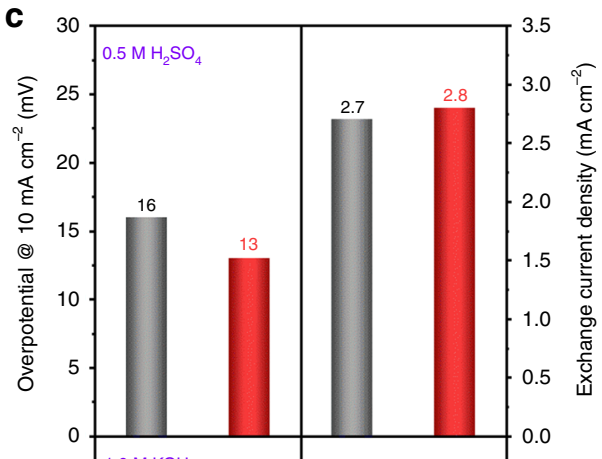

d

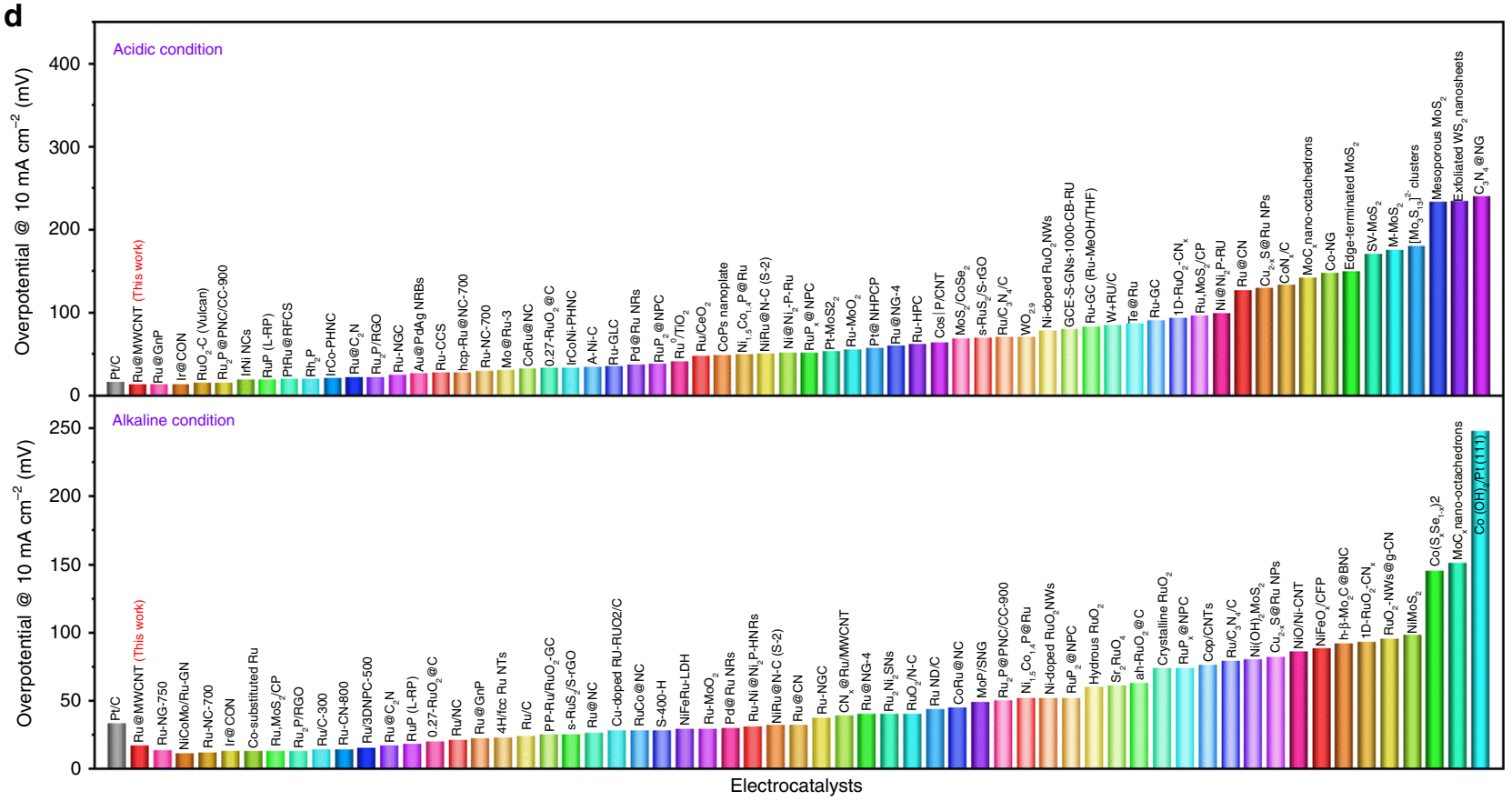

Fig. 3 Electrochemical HER performance of the Ru@MWCNT and Pt/C catalysts. a, b Polarization curves and corresponding Tafel plots in $\mathrm{N}_{2}$-saturated $0.5 \mathrm{M}$ aq. $\mathrm{H}_{2} \mathrm{SO}_{4}$ solution and $1.0 \mathrm{M}$ aq. $\mathrm{KOH}$ solution. Scan rate: $5 \mathrm{mV} \mathrm{s}^{-1}$. c Overpotentials at $10 \mathrm{~mA} \mathrm{~cm}^{-2}$ and exchange current density in $\mathrm{N}_{2}$-saturated $0.5 \mathrm{M}$ aq. $\mathrm{H}_{2} \mathrm{SO}_{4}$ solution and $1.0 \mathrm{M}$ aq. $\mathrm{KOH}$ solution. d Comparison of the overpotentials at $10 \mathrm{~mA} \mathrm{~cm}^{-2}$ with recently reported HER catalysts in both acidic and alkaline conditions.

solution. As references, commercial Pt/C and bare MWCNT were also tested under the same conditions and compared. The MWCNT did not show catalytic activity toward HER in the range of applied potential. On the other hand, both the $\mathrm{Pt} / \mathrm{C}$ and Ru@MWCNT required an overpotential of $\sim 0 \mathrm{mV}$ to induce hydrogen evolution (Fig. 3a).

Notably, the HER current density of Ru@MWCNT sharply increased as the overportential increased, with a Tafel slope of 27 $\mathrm{mV} \mathrm{dec}^{-1}$ similar to $\mathrm{Pt} / \mathrm{C}$ (Fig. 3b). The small Tafel slope indicates that the rate determining step is the recombination of chemisorbed hydrogen, following the Volmer-Tafel mechanism $^{45-47}$.

As a critical parameter for practical evaluation, the overpotential at a current density of $10 \mathrm{~mA} \mathrm{~cm}^{-2}$ was evaluated for each catalyst. Ru@MWCNT displayed an overpotential of $13 \mathrm{mV}$ and $\mathrm{Pt} / \mathrm{C}$ required $16 \mathrm{mV}$ to deliver a current density of $10 \mathrm{~mA}$ $\mathrm{cm}^{-2}$. From the Tafel slope, the exchange current density of Ru@MWCNT was $2.8 \mathrm{~mA} \mathrm{~cm}^{-2}$, which was similar to the $\mathrm{Pt} / \mathrm{C}$ 
(2.7 $\left.\mathrm{mA} \mathrm{cm}^{-2}\right)$, indicating Ru@MWCNT electrode's rapid HER kinetics (Fig. 3c). In acidic conditions, electrochemical impedance spectroscopy (EIS) analysis of the Ru@MWCNT catalyst exhibited a charge transfer resistance of $1.81 \Omega \mathrm{cm}^{2}$ at an overpotential of $35 \mathrm{mV}$, which was lower than the Pt/C $(2.23 \Omega$ $\mathrm{cm}^{2}$ at $35 \mathrm{mV}$ ). This implies fast electron/proton transfer at the interface of the Ru@MWCNT and the electrolyte (Supplementary Fig. 6). This remarkably improved HER performance is believed to be due to favorable charge transfer between the active sites and the working electrode, which is attributed to the highly conductive MWCNT substrate.

The HER efficiencies of the Ru@MWCNT and commercial $\mathrm{Pt} / \mathrm{C}$ catalysts were evaluated in $\mathrm{N}_{2}$-saturated $1.0 \mathrm{M}$ aq. $\mathrm{KOH}$ solution (Fig. 3a). Interestingly, the Ru@MWCNT catalyst

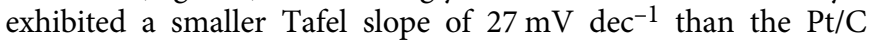
$\left(43 \mathrm{mV} \mathrm{dec}^{-1}\right)$. The smaller Tafel slope indicates that Ru@MWCNT catalyzed the reaction faster than Pt/C (Fig. 3b). The exchange current density $\left(2.4 \mathrm{~mA} \mathrm{~cm}^{-2}\right)$ of the Ru@MWCNT was also higher than the Pt/C $\left(1.4 \mathrm{~mA} \mathrm{~cm}^{-2}\right)$, indicating it had higher electrocatalytic HER activity in alkaline medium (Fig. 3c). As a result, the overpotential required to generate a current density of $10 \mathrm{~mA} \mathrm{~cm}^{-2}$ was only $17 \mathrm{mV}$, smaller than the benchmark Pt/C $(33 \mathrm{mV})$. The charge transfer resistance of the Ru@MWCNT calculated from EIS was $2.38 \Omega$ $\mathrm{cm}^{2}$ at an overpotential of $45 \mathrm{mV}$, while that of $\mathrm{Pt} / \mathrm{C}$ was $4.22 \Omega$ $\mathrm{cm}^{2}$ (Supplementary Fig. 6). The lower charge transfer resistance of Ru@MWCNT also indicates efficient HER charge transfer kinetics compared to $\mathrm{Pt} / \mathrm{C}$ in alkaline conditions.

The overpotentials of Ru@MWCNT at $10 \mathrm{~mA} \mathrm{~cm}^{-2}$ in acidic (Fig. 3d and Supplementary Table 2) and alkaline media (Fig. 3d and Supplementary Table 3) were compared with other HER catalysts reported in recent studies ${ }^{18,25,48-50}$. The substrate, MWCNT, did not show any HER catalytic activity, while Ru@MWCNT exhibited excellent HER performance due to the presence of the small $\mathrm{Ru}$ nanoparticles (average $3.4 \mathrm{~nm}$ ) stably anchored on its surface (Supplementary Fig. 7).

To evaluate the electrochemical surface area (ECSA) of the catalysts, the underpotential deposition of copper (Cu-UPD) on Ru@MWCNT and Pt/C were carried out. The ECSA of Ru@MWCNT was $7996.15 \mathrm{~m}^{2} \mathrm{~g}^{-1}{ }_{\mathrm{Ru}}$, which was approximately two times higher than commercial $\mathrm{Pt} / \mathrm{C} \quad\left(3638.67 \mathrm{~m}^{2} \mathrm{~g}^{-1} \mathrm{Pt}\right)$ (Supplementary Fig. 8).

In order to identify the active sites on the Ru@MWCNT, thiocyanate ions $\left({ }^{-} \mathrm{SCN}\right)$, an active site toxin of metal catalysts, was added to the $0.5 \mathrm{M}$ aq. $\mathrm{H}_{2} \mathrm{SO}_{4}$ electrolyte. The addition of - SCN dramatically reduced the activity of the Ru@MWCNT, indicating that the Ru nanoparticles on the Ru@MWCNT were the active sites for HER catalysis (Supplementary Fig. 9).

To evaluate the long-term stability of Ru@MWCNT and Pt/C catalysts in both $0.5 \mathrm{M}$ aq. $\mathrm{H}_{2} \mathrm{SO}_{4}$ (Figs. $4 \mathrm{a}$ ) and $1.0 \mathrm{M}$ aq. $\mathrm{KOH}$ solutions (Fig. 4b), cyclic stability tests were conducted at a scan rate of $100 \mathrm{mV} \mathrm{s}^{-1}$. In acidic conditions, the commercial $\mathrm{Pt} / \mathrm{C}$ showed an $8 \mathrm{mV}$ negative shift at a current density of $10 \mathrm{~mA} \mathrm{~cm}^{-2}$, while the Ru@MWCNT catalyst displayed only a $4 \mathrm{mV}$ negative shift after 10,000 cycles. In alkaline conditions, the Ru@MWCNT exhibited 20 times better electrochemical stability than $\mathrm{Pt} / \mathrm{C}$ (Fig. 4c). Stability was also examined via chronoamperometry technique at the applied potentials 20 and $35 \mathrm{mV}$, respectively, in acidic and alkaline media for $50 \mathrm{~h}$, and the Ru@MWCNT exhibited no apparent loss in current density compared to $\mathrm{Pt} / \mathrm{C}$ (Supplementary Fig. 10). In addition, TEM images of the Ru@MWCNT after the long-term stability test showed no change in morphology (Supplementary Fig. 11). These results indicate the exceptional stability of Ru@MWCNT compared to commercial Pt/C in both acidic and alkaline media.
For a fair comparison of catalytic activity, the polarization curves of Ru@MWCNT and Pt/C were normalized by ECSA. In $0.5 \mathrm{M}$ aq. $\mathrm{H}_{2} \mathrm{SO}_{4}$ solution, the $\mathrm{Ru} @ M W C N T$ showed slightly higher specific activity than $\mathrm{Pt} / \mathrm{C}$ for a series of overpotentials (Fig. 4d). A more dramatic difference was observed in the specific activity between Ru@MWCNT $\left(0.315 \mathrm{~mA} \mathrm{~cm}^{-2}\right)$ and $\mathrm{Pt} / \mathrm{C}$ $\left(0.122 \mathrm{~mA} \mathrm{~cm}^{-2}\right)$ in $1.0 \mathrm{M}$ aq. $\mathrm{KOH}$ solution (Fig. $\left.4 \mathrm{~d}\right)$. At an overpotential of $30 \mathrm{mV}$, the Ru@MWCNT showed 2.5 times higher specific activity than the $\mathrm{Pt} / \mathrm{C}$. This result indicates superior inherent catalytic activity, which is associated with the stronger $\mathrm{H}_{2} \mathrm{O}$ binding energy and faster $\mathrm{H}_{2} \mathrm{O}$ dissociation at the surface of the Ru nanoparticles on the Ru@MWCNT catalyst ${ }^{25}$. As a result, Ru@MWCNT can supply protons faster for more efficient hydrogen generation. Given its fast proton adsorption and reduction via appropriate hydrogen bond energy, fast proton supply, and rapid release of product $\left(\mathrm{H}_{2}\right)$, the Ru@MWCNT is a highly active HER catalyst.

To compare and evaluate the HER performance of the catalyst, we evaluated its turnover frequency (TOF), which is an important criterion for HER catalysts. TOF is the basis for determining inherent electrocatalytic efficiency, and the overpotential at 10 $\mathrm{mA} \mathrm{cm}{ }^{-2}$ predicts the actual HER applicability. The TOF values for the active sites of the catalysts were calculated under acidic and alkaline conditions, following the previously reported method 22,23 . In $0.5 \mathrm{M}$ aq. $\mathrm{H}_{2} \mathrm{SO}_{4}$ solution, the TOF value of $\mathrm{Ru@MWCNT} \mathrm{at} 25 \mathrm{mV}$ was $0.70 \mathrm{H}_{2} \mathrm{~s}^{-1}$, which is very competitive compared to $\mathrm{Pt} / \mathrm{C}\left(0.67 \mathrm{H}_{2} \mathrm{~s}^{-1}\right.$ at $\left.25 \mathrm{mV}\right)$ and other reported HER catalysts (Fig. 4e and Supplementary Table 4). In addition, in an alkaline solution, the TOF value of Ru@MWCNT at $25 \mathrm{mV}$ was $0.40 \mathrm{H}_{2} \mathrm{~s}^{-1}$, which is higher than that of $\mathrm{Pt} / \mathrm{C}(0.25$ $\mathrm{H}_{2} \mathrm{~s}^{-1}$ ) (Fig. 4e and Supplementary Table 5). The TOF values of the reference $\mathrm{Pt} / \mathrm{C}$ are also reliable compared to other HER catalysts reported in recent studies (Supplementary Tables 4, 5). Hence, the TOF values in both conditions indicate that Ru@MWCNT outperforms Pt/C HER activity.

To further examine the catalysts from different perspectives, the mass activity of each catalyst was evaluated by normalizing the polarization curves with the masses of $\mathrm{Ru}$ and Pt. Mass activity is closely related to cost for practical applications. As shown in Fig. $4 \mathrm{f}$, at the overpotential of $20 \mathrm{mV}$, the mass activity of Ru@MWCNT was $380 \mathrm{~mA} \mathrm{mg}^{-1}{ }_{\mathrm{Ru}}$ in acidic medium and 186 $\mathrm{mA} \mathrm{mg}{ }^{-1}{ }_{\mathrm{Ru}}$ in alkaline medium. These values were much higher than $\mathrm{Pt} / \mathrm{C}$ (165 and $52 \mathrm{~mA} \mathrm{mg}^{-1}{ }_{\mathrm{Pt}}$, respectively). Therefore, it can be safely stated that Ru@MWCNT has significant advantages over $\mathrm{Pt} / \mathrm{C}$ in terms of overall catalytic performance and cost.

The full water-splitting system analysis. To further demonstrate an advanced practical use of Ru@MWCNT for water-splitting using an alkaline electrolyte (1.0 $\mathrm{M}$ aq. $\mathrm{KOH}$ solution), twoelectrode devices with oxygen and hydrogen evolution electrodes were fabricated. The carbon papers (CPs) used as substrates for the electrodes were coated with the catalysts by electrospray (Supplementary Fig. 12). Both Ru@MWCNT and Pt/C coated on $\mathrm{CP}$, as well as bare $\mathrm{CP}$, was tested as a HER electrode. As the oxygen evolution reaction (OER) electrode, commercial iridium oxide $\left(\mathrm{IrO}_{2}\right)$ was coated on the CP. In order to accurately determine the actual amount of hydrogen generation, a systematic experiment was conducted by connecting a closed watersplitting device (HER + OER) directly to a gas chromatography (GC) instrument (Fig. 5a). The area of each electrode was $1 \mathrm{~cm}^{2}$. Prior to the two-electrode evaluation, a three-electrode experiment was conducted to confirm the HER performance of the prepared electrodes. Current densities of devices with different HER electrodes were obtained (Fig. 5b). The Ru@MWCNT 
a

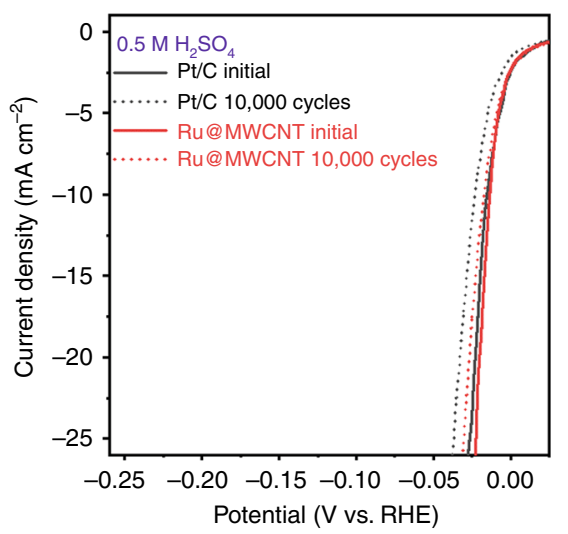

d

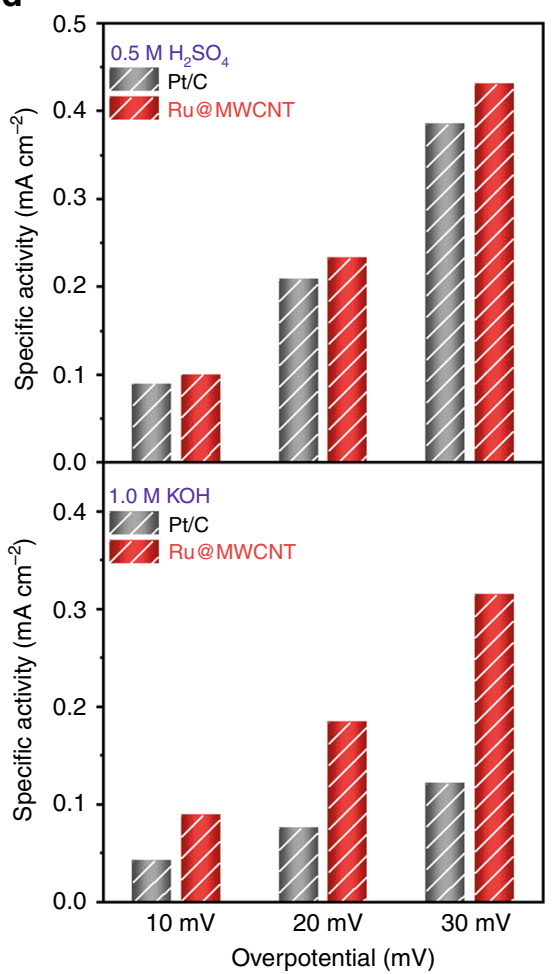

b

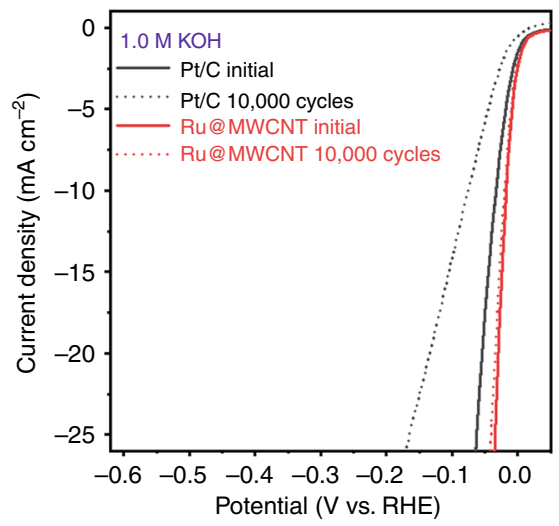

e

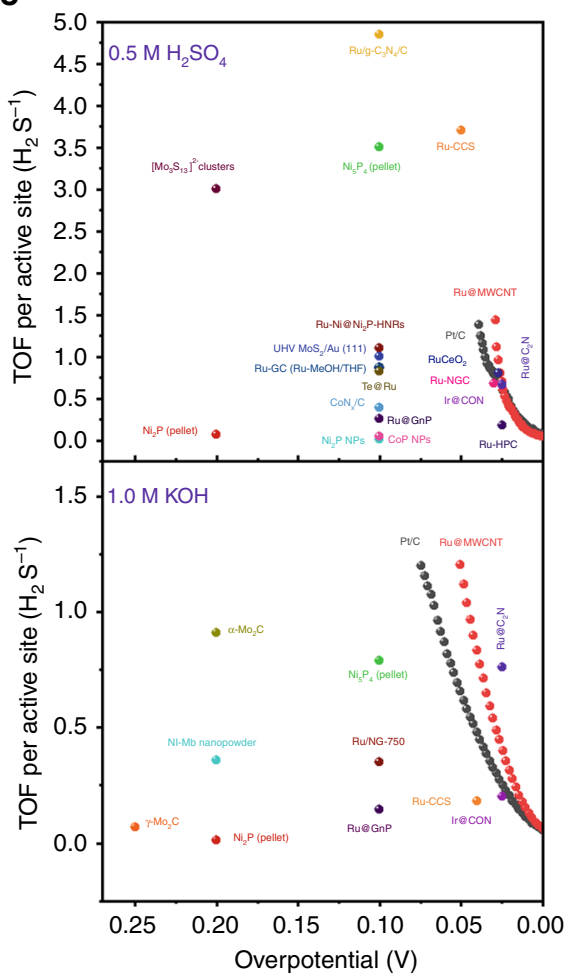

C

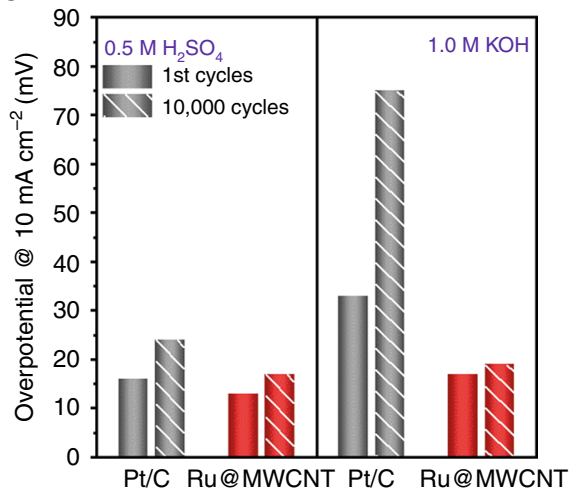

f

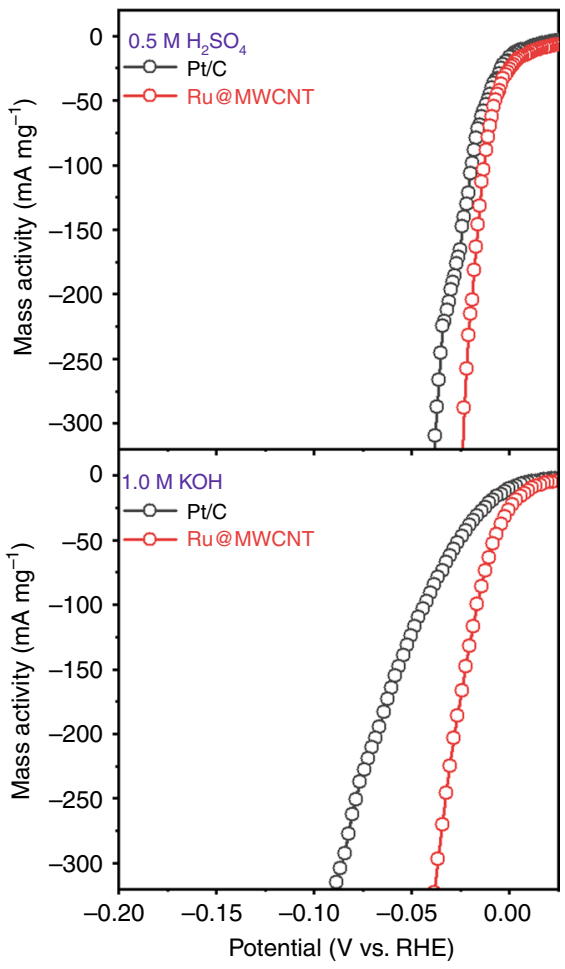

Fig. 4 Comparison of electrochemical HER parameters. a, b The polarization curves were recorded before and after 10,000 CV potential cycles.

c Comparison of overpotential changes at $10 \mathrm{~mA} \mathrm{~cm}^{-2}$ after $10,000 \mathrm{CV}$ potential cycles in acidic and alkaline conditions. $\mathbf{d}$ Specific activities at different overpotentials $\left(10,20\right.$, and $30 \mathrm{mV}$ ) in $\mathrm{N}_{2}$-saturated acidic and alkaline conditions, respectively. e Comparison of TOF values of the Ru@MWCNT and Pt/C with other recently reported HER catalysts in acidic and alkaline conditions, respectively. $\mathbf{f}$ Mass activities in $\mathrm{N}_{2}$-saturated $0.5 \mathrm{M}^{2}$ aq. $\mathrm{H}_{2} \mathrm{SO}_{4}$ and $1.0 \mathrm{M}$ aq. $\mathrm{KOH}$ solutions, respectively.

electrode showed overpotentials of 10.4, 19.4, and 28.4 at 10, 20, and $30 \mathrm{~mA} \mathrm{~cm}^{-2}$, respectively, while the $\mathrm{Pt} / \mathrm{C}$ electrode showed 26.4, 40.4, and $50.4 \mathrm{mV}$ at each corresponding current density.

A constant current was applied to the system for $20 \mathrm{~h}$ and the amount of hydrogen generated was measured every hour. As shown in Fig. $5 \mathrm{c}-\mathrm{e}$, the hydrogen production of the Ru@MWCNT per voltage was 2222.3, 3221.9, and $4194.0 \mu \mathrm{mol} \mathrm{V} \mathrm{V}^{-1}$ meaning it produced $15.6 \%$ more than the Pt/C (Supplementary Tables 6-8). In addition, the hydrogen production of Ru@MWCNT per power consumption was also $15.4 \%$ higher than the Pt/C (Fig. $5 \mathrm{f}$ and Supplementary Table 9). Faradaic efficiency was also determined in the range of 1.5-1.8 V (Fig. 5g). The bare CP showed a Faradaic efficiency of only $11.4 \%$ at $1.8 \mathrm{~V}$ and there was no HER activity in the range of $1.5-1.7 \mathrm{~V}$. The $\mathrm{Pt} / \mathrm{C}$ electrode showed Faradaic efficiencies of $46.99,81.98,85.88$, and $85.97 \%$ at $1.5,1.6,1.7$, and
$1.8 \mathrm{~V}$, respectively, while the Ru@MWCNT electrode showed $85.88,87.31,92.24$, and $92.28 \%$ at each corresponding voltage. Once again, the results indicated that the Ru@MWCNT catalyst was superior to the benchmark Pt/C (Supplementary Table 9).

The Ru@MWCNT catalyst was also coated on a large size titanium (Ti) mesh type electrode to check the practical application of the catalyst (Supplementary Video 1).

Active site identification by DFT calculations. First-principle density functional theory (DFT) calculations were also performed to gain more insight into the enhanced electrocatalytic activity of Ru@MWCNT active sites for hydrogen evolution reaction. It is widely known that the formation energy of metal-hydrogen $(\mathrm{M}-\mathrm{H})$ bond plays an important role in hydrogen evolution. Being at the center of volcano plot for electrocatalysts, Pt displays 
a

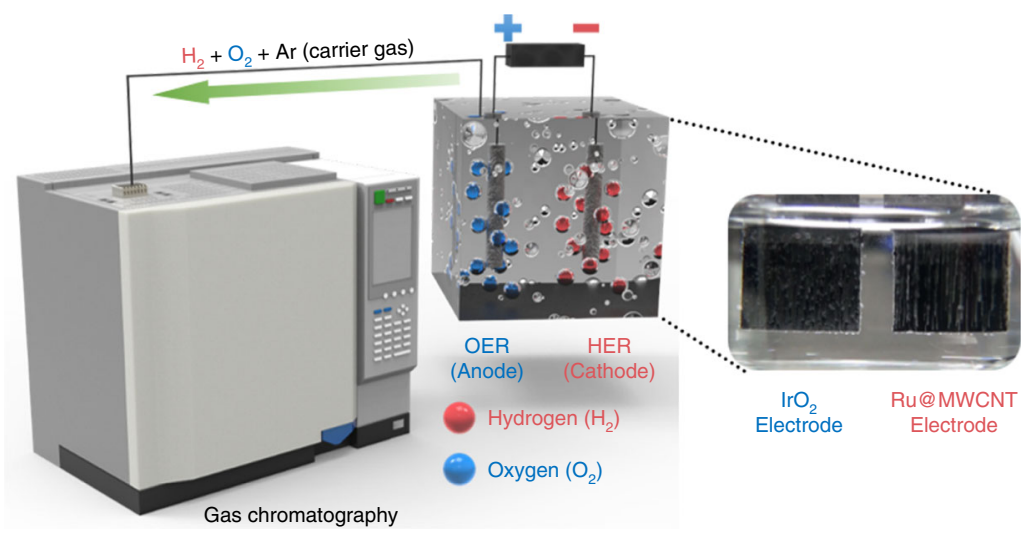

C

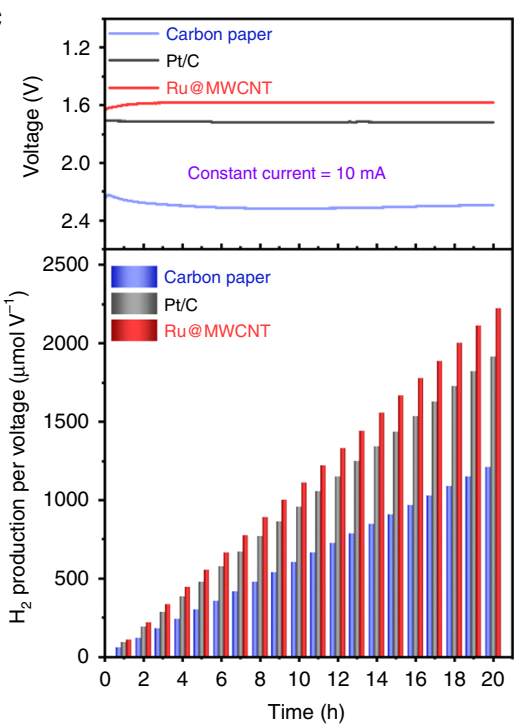

d

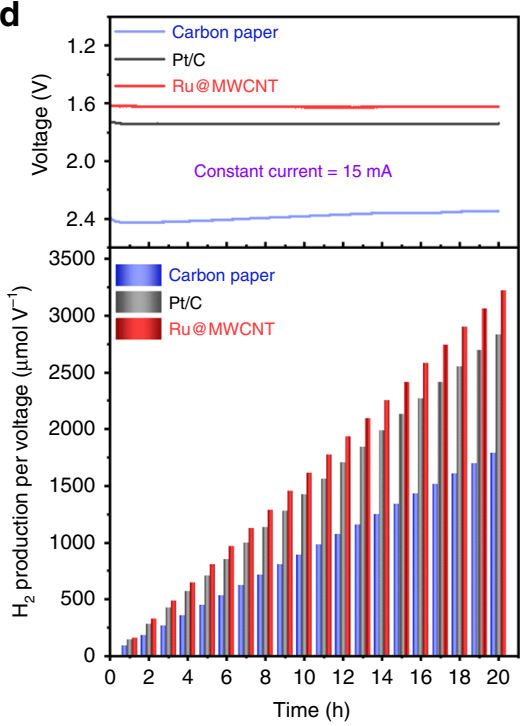

b

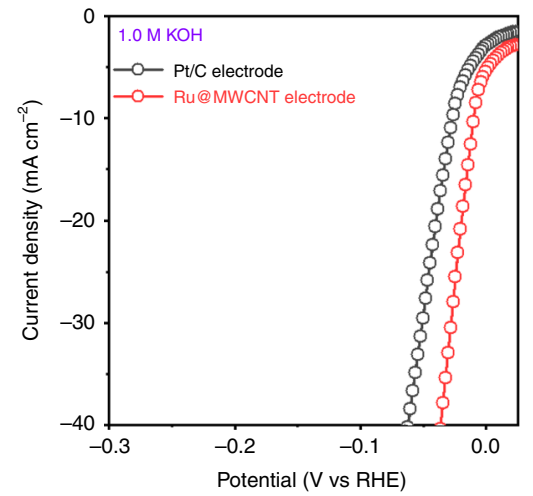

e

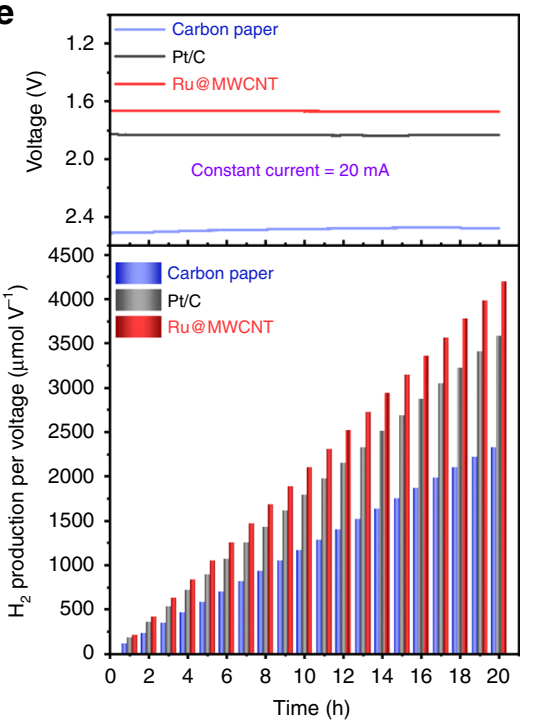

$\mathbf{f}$

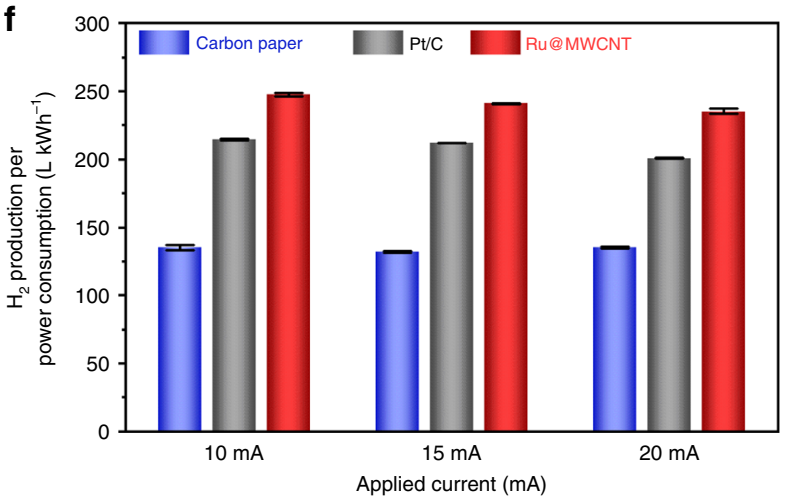

g

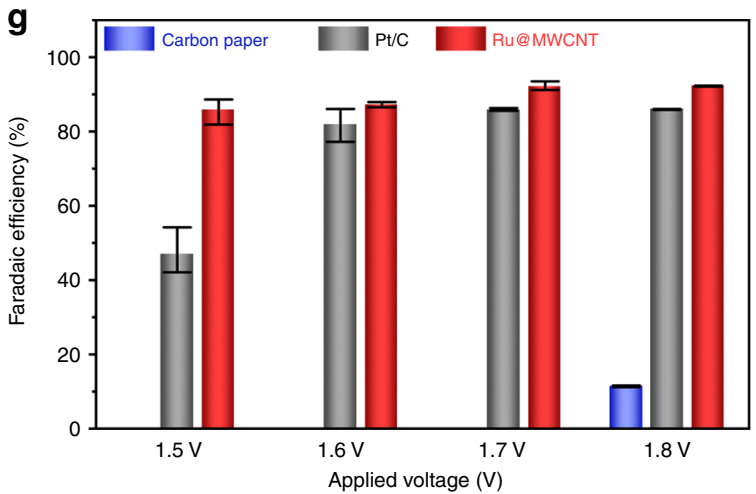

Fig. 5 HER performance evaluation in actual water splitting. a Schematic diagram of the two-electrode system measurement. $\mathbf{b}$ Polarization curves in $\mathrm{N}_{2}{ }^{-}$ saturated $1.0 \mathrm{M}$ aq. $\mathrm{KOH}$ solution. Scan rate: $5 \mathrm{mV} \mathrm{s}^{-1}$. c-e The voltage changes at constant current and the corresponding hydrogen production per voltage at specific currents of 10, 15, and $20 \mathrm{~mA}$. $\mathbf{f}$ Hydrogen production per power consumption at specific current (10, 15, and $20 \mathrm{~mA})$. The error bar reflects the three device results. $\mathbf{g}$ Faradaic efficiency at a specific voltage $(1.5,1.6,1.7$, and $1.8 \mathrm{~V})$. The error bar reflects the three device results.

the optimal $\mathrm{M}-\mathrm{H}$ binding energy, which is neither too weak nor too strong ${ }^{25}$. Catalysts having $\mathrm{M}-\mathrm{H}$ binding energy similar or close to $\mathrm{Pt}-\mathrm{H}(0.53 \mathrm{eV})$ will efficiently promote hydrogen evolution. The DFT calculations were performed based on previously reported Ru@ $\mathrm{C}_{2} \mathrm{~N}$ for a clear comparison ${ }^{25}$. To sustain the catalytic activity of $\mathrm{Ru}$ nanoparticles, the important point is to prevent their aggregation (Ostwald ripening). The calculation showed that Ru nanoparticles on Ru@MWCNT have closer Pt
$-\mathrm{H}$ binding energy than on Ru@ $\mathrm{C}_{2} \mathrm{~N}$ (Supplementary Fig. 14). This result indicates that Ru@MWCNT can have better HER performance than $\mathrm{Ru} @ \mathrm{C}_{2} \mathrm{~N}$. For more details, hydrogen binding energies of possible $\mathrm{H}$ binding sites are identified, and the four most stable energies are 0.58, 0.64, 0.64, and $0.62 \mathrm{eV}$ (Supplementary Fig. 15). All stable configurations of Ru@MWCNT show lower energies than $\mathrm{Ru}_{0} \mathrm{C}_{2} \mathrm{~N} \quad(0.68 \mathrm{eV})$, suggesting that Ru@MWCNT can display enhanced catalytic activity. An 
important point to be noted is that the Ru@MWCNT has an energy of $-5.23 \mathrm{eV}$ (10 Ru-C bonds) (Supplementary Fig. 16), implying that there are strong $\mathrm{Ru}-\mathrm{C}$ bonds between $\mathrm{Ru}$ nanoparticles and MWCNT. This result reflects the stability of Ru nanoparticles on the surface of MWCNT (Ru@MWCNT) during long cycling test. Furthermore, the formation of $\mathrm{Ru}-\mathrm{C}$ bonds was confirmed by EXAFS results (Supplementary Fig. 1), supporting that the aggregation (Ostwald ripening) of $\mathrm{Ru}$ nanoparticles can be hampered by forming strong bonds between Ru and MWCNT.

\section{Discussion}

In summary, we have developed an efficient and stable HER catalyst for both acidic and alkaline media via a simple synthesis route. The catalyst consists of ruthenium $(\mathrm{Ru})$ nanoparticles uniformly distributed and anchored on the surface of multiwalled carbon nanotubes (MWCNTs), or (Ru@MWCNT). The catalyst was realized by the formation of ruthenium carboxylate complexes, created between $\mathrm{Ru}$ ions $\left(\mathrm{Ru}^{3+}\right)$ and MWCNT-COOH, which was produced by the partial oxidation of MWCNT in nitric acid. Subsequent chemical $\left(\mathrm{NaBH}_{4}\right)$ and thermal reductions (annealing) turned the $\mathrm{Ru}^{3+}$ ions into $\mathrm{Ru}^{0}$ nanoparticles on the surface of MWCNT. The smaller particle size distribution and particle uniformity supports higher mass activity. MWCNT, which is widely known as a conductive material, not only provided an efficient catalytic support, stably anchoring the $\mathrm{Ru}$ nanoparticle active sites, but also fast electron transport.

As a result, the overall HER performance of the Ru@MWCNT, in terms of overpotential at $10 \mathrm{~mA} \mathrm{~cm}^{-2}$, Tafel slope, and longterm stability, was superior to commercial $\mathrm{Pt} / \mathrm{C}$ in both acidic and alkaline media. Regarding its potential value in practical applications, the Ru@MWCNT also displayed higher mass activity than commercial Pt/C. Most importantly, Ru@MWCNT has strong potential for mass production at low-cost, making it advantageous for use in practical applications. Last but not least, in an actual water-splitting experiment, Ru@MWCNT demonstrated an average Faradaic efficiency of $92.28 \%$ at $1.8 \mathrm{~V}$, resulting in $15.4 \%$ higher hydrogen production per power consumption than $\mathrm{Pt} / \mathrm{C}$.

\section{Method \\ Oxidation of multiwalled carbon nanotubes (MWCNT) ${ }^{51-54}$. In a three-neck round bottom flask, MWCNT (10 g, CM-95, Hanhwa Nanotech Co.) was dispersed in concentrated nitric acid $(500 \mathrm{~mL})$ after sonication for $1 \mathrm{~h}$. Then, the reaction flask was placed in an oil bath and heated under reflux for $24 \mathrm{~h}$. After cooling down to room temperature, the reaction mixture was poured into deionized water $(1 \mathrm{~L})$ and the precipitates, oxidized MWCNT, were collected by suction filtration. The product was further Soxhlet extracted with water and methanol to completely remove residual acid and other impurities, if any. The sample was finally freeze- dried for 3 days at $-120^{\circ} \mathrm{C}$ under reduced pressure.}

Preparation of Ru@MWCNT. Partially oxidized MWCNTs (MWCNT-COOH, $10.0 \mathrm{~g})$ and ruthenium chloride $\left(\mathrm{RuCl}_{3}, 3.0 \mathrm{~g}\right)$ were dispersed in $\mathrm{N}$-methyl-2-pyrrolidone (NMP, 1.3 L). The mixture was agitated in a sonication bath for $3 \mathrm{~h}$ and further stirred overnight using a magnetic stirrer. The dispersed mixture was further sonicated for $2 \mathrm{~h}$. Then, sodium borohydride ( $10 \%$ solution in NMP, $60 \mathrm{~mL}$ ) was added using a dropping funnel under vigorous stirring. The mixture was stirred for $1 \mathrm{~h}$ and then mixed with acetone $(1.5 \mathrm{~L})$. The precipitates were collected by suction filtration and washed with water and freeze dried at $-120^{\circ} \mathrm{C}$ under reduced pressure for 3 days. The sample was annealed at different temperatures $(600,700$, and $800{ }^{\circ} \mathrm{C}$ ) under argon atmosphere for $2 \mathrm{~h}$ each. After annealing, the samples were further washed with water to remove unbound metal impurities in the matrix, if any. Finally, the samples were dried under reduced pressure.

Electrochemical characterizations. The electrochemical studies were carried out on an electrochemical workstation (Ivium, Netherlands) with a typical threeelectrode cell. A graphite rod and an $\mathrm{Ag} / \mathrm{AgCl}$ (saturated $\mathrm{KCl}$ ) electrode were used as the counter electrode and reference electrode, respectively. All potentials were referenced with a reversible hydrogen electrode (RHE). Each catalyst (5 mg) was dispersed with Nafion $(20 \mu \mathrm{L}, 5 \mathrm{wt} \%$ in a mixture of lower aliphatic alcohol and water, Aldrich Chemical Inc.) in isopropyl alcohol $(1.0 \mathrm{~mL})$. The mixture was sonicated for $30 \mathrm{~min}$ in an ice bath to form a uniform catalyst ink. The ink was drop casted onto a rotating ring-disk electrode ( $4 \mathrm{~mm}$ in diameter, RRDE) to form a film for the electrochemical tests. The loading amounts of each catalyst were 0.70 and $0.16 \mathrm{mg} \mathrm{cm}^{-2}$ for the acidic and alkaline media, respectively. Linear sweep voltammetry (LSV) was conducted in both $0.5 \mathrm{M}$ aq. $\mathrm{H}_{2} \mathrm{SO}_{4}$ and $1.0 \mathrm{M}$ aq. $\mathrm{KOH}$ solutions at a scan rate of $5 \mathrm{mV} \mathrm{s}^{-1}$. The solution resistances $\left(\mathrm{R}_{\mathrm{s}}\right)$ in the $0.5 \mathrm{M}$ aq $\mathrm{H}_{2} \mathrm{SO}_{4}$ and $1.0 \mathrm{M}$ aq. $\mathrm{KOH}$ solutions were 15 and $17 \Omega$, respectively, tested by Nyquist plots. All data were further used for the Ohmic drop (iR) correction. The reference electrode was calibrated, and all potentials were referenced to a RHE (Supplementary Fig. 13).

Active sites calculations. The underpotential deposition (UPD) of copper ( $\mathrm{Cu}$ ) was used to calculate the active sites of the Ru@MWCNT and Pt/C. In this method, the number of active sites (n) can be calculated based on the UPD copper stripping charge $\left(\mathrm{Q}_{\mathrm{Cu}}, \mathrm{Cu}_{\text {upd }} \rightarrow \mathrm{Cu}^{2+}+2 \mathrm{e}^{-}\right)$using the following equation ${ }^{25}$.

$\mathrm{n}=\mathrm{Q}_{\mathrm{Cu}} / 2 \mathrm{~F}$

where $\mathrm{F}$ is the Faraday constant $\left(96,485.3 \mathrm{C} \mathrm{mol}^{-1}\right)$.

Measurement of the turnover frequency (TOF). The TOF $\left(\mathrm{s}^{-1}\right)$ was calculated with the following equation.

$\mathrm{TOF}=\mathrm{I} /(2 \mathrm{Fn})$

where $\mathrm{I}$ is the current (A) during linear sweep voltammetry (LSV), F is the Faraday constant $\left(96485.3 \mathrm{C} \mathrm{mol}^{-1}\right), \mathrm{n}$ is the number of active sites (mol). The factor $1 / 2$ is based on the assumption that two electrons are necessary to form on hydrogen molecules.

Ru@MWCNT was first polarized at 0.22, 0.23, 0.24, 0.25, 0.26, 0.27, 0.28, 0.29, 0.30 , and $0.31 \mathrm{~V}$ for $100 \mathrm{~s}$ (Supplementary Fig. $8 \mathrm{a}$ ). For the given polarization potential, there were only two oxidation peaks related to bulk and monolayer of $\mathrm{Cu}$. To obtain monolayer of copper, $0.26 \mathrm{~V}$ was selected in the following test for Ru@MWCNT (Supplementary Fig. 8b, c).

Preparation of HER electrodes. Each catalyst ( $5 \mathrm{mg}, \mathrm{Ru} @ M W C N T, \mathrm{Pt} / \mathrm{C}$ or $\mathrm{IrO}_{2}$ ) was dispersed in isopropyl alcohol $(1.0 \mathrm{~mL})$ after applying sonication for $30 \mathrm{~min}$. The resultant catalyst ink was directly deposited onto carbon paper (CP) using an electrospray method. First, each catalyst ink was placed into a plastic syringe equipped with a 30-gauge stainless steel hypodermic needle. The needle was connected to a high voltage power supply (ESN-HV30). A voltage of $\sim 4.3 \mathrm{kV}$ was applied between a metal orifice and the conducting substrate at a distance of $8 \mathrm{~cm}$. The feed rate was controlled by the syringe pump (KD Scientific Model 220) at a constant flow rate of $20 \mu \mathrm{L} \mathrm{min}{ }^{-1}$. The electric field overcomes the surface tension of the droplets, resulting in the minimization of numerous charged mists. Each electrode was tested after drying in vacuum oven at room temperature for 1 day.

Computation method. To simulate the experimental results, an icosahedral symmetric $\mathrm{Ru}_{13}$ nanoparticle is attached to wall of carbon nanotube (CNT). The Vienna Ab initio Simulation Package (VASP) calculations are carried out to obtain the ground state of many electrons system in the framework of density functional theory ${ }^{55-57}$. The plane-wave basis set with an energy cutoff of $500 \mathrm{eV}$ and the PBEtype gradient-corrected exchange-correlation potential suggested by Perdew, Burke, and Ernzerhof were employed ${ }^{58}$. The ionic potentials were described by the projector-augmented wave potentials, and the atomic configurations were selectively relaxed with the residual forces smaller than $0.001 \mathrm{eV} / \AA^{56}$. Periodic boundary conditions for DWCNT are made by $20 \AA \times 25 \AA \times 14.85 \AA$ unit cell and $1 \times 1 \times 6$ kpoints sampling. In order to reduce the calculation cost, we cut the fully optimized DWCNT into half and get a semi-cylinder shape with 108 carbon atoms. The carbons further from $\mathrm{Ru}_{13}$ nanoparticle bonding region are fixed and all the other atoms are relaxed in geometric optimization.

Materials characterizations. The morphologies of the samples were studied by FE-SEM (Nanonova 230, FEI, USA) and high-resolution transmission electron microscopy (HR-TEM, JEM-2100F, JEOL, Japan). Specific surface area was determined by nitrogen adsorption-desorption isotherms, using the BET method (BELSORP-max, BEL, Japan). Thermogravimetric analysis (TGA) was performed at a ramping rate of $10^{\circ} \mathrm{C} \mathrm{min}{ }^{-1}$ in air on a thermogravimetric analyzer (Q200, TA, USA). X-ray diffraction (XRD) patterns were recorded on a high-power X-ray diffractometer (D/MAZX $2500 \mathrm{~V} / \mathrm{PC}$, Rigaku, Japan), using $\mathrm{Cu}-\mathrm{Ka}$ radiation (35 $\mathrm{kV}, 20 \mathrm{~mA}, \lambda=1.5418 \AA$ ). An X-ray photoelectron spectrometer (XPS, K-alpha, Thermo Fisher Scientific, UK) and elemental analysis (EA, Flash 2000 Analyzer) were employed to determine chemical composition. The electrochemical HER test was initiated, and the evolved hydrogen gas was analyzed by gas chromatography (GC-2010 Plus, Shimadzu, Japan), with a thermal conductivity detector (TCD). Argon was used as the carrier gas. X-ray absorption fine spectra of the prepared catalysts were collected in the transmission mode using ionization detectors (Oxford) at the Pohang Accelerator Laboratory (PAL). The X-ray absorption spectra for the Ru K edge were acquired at room temperature using beamline $6 \mathrm{D}$ of $\mathrm{PAL}$, where their X-ray energies from the EXAFS analysis were calibrated with $\mathrm{Ru}$ foil. Background subtraction, normalization and Fourier transformation (FT) were done by standard procedures with ATHENA program. The extracted EXAFS signal, $\chi(\mathrm{r})$ and $\mathrm{k}^{2} \chi(\mathrm{k})$ were analyzed for all samples. The selected $k$ ranges for $\mathrm{Ru}$ 
acetylacetonate, Ru@MWCNT (before heat-treatment) and Ru@MWCNT (after heat-treatment) in plotting the Ru K-edge graphs were 3.0-11.0, 3.0-11.0, and $3.0-8.7$, respectively, and the selected $\mathrm{R}$ range is $1.0-3.0$ for all samples.

\section{Data availability}

The data supporting this study are available from the corresponding author upon reasonable request.

Received: 9 October 2019; Accepted: 12 February 2020;

Published online: 09 March 2020

\section{References}

1. Kibsgaard, J., Chen, Z., Reinecke, B. N. \& Jaramillo, T. F. Engineering the surface structure of $\mathrm{MoS}_{2}$ to preferentially expose active edge sites for electrocatalysis. Nat. Mater. 11, 963-969 (2012).

2. Turner, J. A. Sustainable hydrogen production. Science 305, 972-974 (2004).

3. Morales-Guio, C. G., Stern, L.-A. \& Hu, X. Nanostructured hydrotreating catalysts for electrochemical hydrogen evolution. Chem. Soc. Rev. 43, 6555-6569 (2014).

4. Jiao, Y., Zheng, Y., Jaroniec, M. \& Qiao, S. Z. Design of electrocatalysts for oxygen- and hydrogen-involving energy conversion reactions. Chem. Soc. Rev. 44, 2060-2086 (2015).

5. Zou, X. \& Zhang, Y. Noble metal-free hydrogen evolution catalysts for water splitting. Chem. Soc. Rev. 44, 5148-5180 (2015).

6. Mazloomi, K. \& Gomes, C. Hydrogen as an energy carrier: prospects and challenges. Renew. Sustain. Energy Rev. 16, 3024-3033 (2012).

7. Norskov, J. K. \& Christensen, C. H. Toward efficient hydrogen production at surfaces. Science 312, 1322-1323 (2006).

8. Vesborg, P. C. K., Seger, B. \& Chorkendorff, I. Recent development in hydrogen evolution reaction catalysts and their practical implementation. $J$. Phys. Chem. Lett. 6, 951-957 (2015).

9. Li, M. et al. Pt monolayer coating on complex network substrate with high catalytic activity for the hydrogen evolution reaction. Sci. Adv. 1, el400268 (2015).

10. Wang, J., Xu, F., Jin, H., Chen, Y. \& Wang, Y. Non-noble metal-based carbon composites in hydrogen evolution reaction: fundamentals to applications. Adv. Mater. 29, 1605838 (2017).

11. Faber, M. S. et al. High-performance electrocatalysis using metallic cobalt pyrite $\left(\cos _{2}\right)$ micro- and nanostructures. J. Am. Chem. Soc. 136, 10053-10061 (2014).

12. Wang, W. et al. Self-templating construction of hollow amorphous $\mathrm{CoMoS}_{4}$ nanotube array towards efficient hydrogen evolution electrocatalysis at neutral pH. Chem. Eur. J. 23, 12718-12723 (2017).

13. Xie, J. et al. Defect-rich $\mathrm{MoS}_{2}$ ultrathin nanosheets with additional active edge sites for enhanced electrocatalytic hydrogen evolution. Adv. Mater. 25, 5807-5813 (2013).

14. Yan, Y., Xia, B., Xu, Z. \& Wang, X. Recent development of molybdenum sulfides as advanced electrocatalysts for hydrogen evolution reaction. ACS Catal. 4, 1693-1705 (2014).

15. McKone, J. R. et al. Evaluation of $\mathrm{Pt}, \mathrm{Ni}$, and $\mathrm{Ni}-\mathrm{Mo}$ electrocatalysts for hydrogen evolution on crystalline Si electrodes. Energy Environ. Sci. 4, 3573-3583 (2011).

16. Ye, S. et al. Highly stable single Pt atomic sites anchored on aniline-stacked graphene for hydrogen evolution reaction. Energy Environ. Sci. 12, 1000-1007 (2019).

17. Zadick, A., Dubau, L., Sergent, N., Berthomé, G. \& Chatenet, M. Huge instability of $\mathrm{Pt} / \mathrm{C}$ catalysts in alkaline medium. ACS Catal. 5, 4819-4824 (2015).

18. Pu, Z., Amiinu, I. S., Kou, Z., Li, W. \& Mu, S. RuP ${ }_{2}$-based catalysts with platinum-like activity and higher durability for the hydrogen evolution reaction at all $\mathrm{pH}$ values. Angew. Chem. Int. Ed. 56, 11559-11564 (2017).

19. Das, R. K. et al. Extraordinary hydrogen evolution and oxidation reaction activity from carbon nanotubes and graphitic carbons. ACS Nano $\mathbf{8}$, $8447-8456$ (2014)

20. Ren, X. et al. In situ electrochemical development of copper oxide nanocatalysts within a TCNQ nanowire array: a highly conductive electrocatalyst for the oxygen evolution reaction. Chem. Commun. $\mathbf{5 4}$ $1425-1428$ (2018).

21. Gong, M., Wang, D.-Y., Chen, C.-C., Hwang, B.-J. \& Dai, H. A mini review on nickel-based electrocatalysts for alkaline hydrogen evolution reaction. Nano Res. 9, 28-46 (2016)

22. Ren, X. et al. Self-supported $\mathrm{CoMoS}_{4}$ nanosheet array as an efficient catalyst for hydrogen evolution reaction at neutral pH. Nano Res. 11, 2024-2033 (2018).

23. Mahmood, J., Anjum, M. A. R. \& Baek, J.-B. Fused aromatic network structures as a platform for efficient electrocatalysis. Adv. Mater. 31, 1805062 (2019).
24. Mahmood, J. et al. Encapsulating iridium nanoparticles inside a $3 \mathrm{~d}$ cage-like organic network as an efficient and durable catalyst for the hydrogen evolution reaction. Adv. Mater. 30, 1805606 (2018).

25. Mahmood, J. et al. An efficient and $\mathrm{pH}$-universal ruthenium-based catalyst for the hydrogen evolution reaction. Nat. Nanotechnol. 12, 441-446 (2017).

26. Ye, R. et al. High performance electrocatalytic reaction of hydrogen and oxygen on ruthenium nanoclusters. ACS Appl. Mater. Interfaces 9, 3785-3791 (2017).

27. Greeley, J. \& Mavrikakis, M. Alloy catalysts designed from first principles. Nat. Mater. 3, 810-815 (2004)

28. Karlberg, G. S. Adsorption trends for water, hydroxyl, oxygen, and hydrogen on transition-metal and platinum-skin surfaces. Phys. Rev. B 74, 153414 (2006).

29. Seh, Z. W. et al. Combining theory and experiment in electrocatalysis: Insights into materials design. Science 355, eaad4998 (2017).

30. Greeley, J., Jaramillo, T. F., Bonde, J., Chorkendorff, I. \& Nørskov, J. K. Computational high-throughput screening of electrocatalytic materials for hydrogen evolution. Nat. Mater. 5, 909-913 (2006).

31. Parsons, R. \& Bockris, J. O. M. Calculation of the energy of activation of discharge of hydrogen ions at metal electrodes. Trans. Faraday Soc. 47, 914-928 (1951).

32. Rüetschi, P. \& Delahay, P. Hydrogen overvoltage and electrode material. a theoretical analysis. J. Chem. Phys. 23, 195-199 (1955).

33. Conway, B. E. \& Bockris, J. O. M. Electrolytic hydrogen evolution kinetics and its relation to the electronic and adsorptive properties of the metal. J. Chem. Phys. 26, 532-541 (1957).

34. Conway, B. E. \& Bockris, J. O. M. Heats of activation in electrode processesthe electrochemical desorption mechanism of the discharge of hydroxonium ions. Can. J. Chem. 35, 1124-1136 (1957).

35. Parsons, R. The rate of electrolytic hydrogen evolution and the heat of adsorption of hydrogen. Trans. Faraday Soc. 54, 1053-1063 (1958).

36. Thomas, J. G. N. Kinetics of electrolytic hydrogen evolution and the adsorption of hydrogen by metals. Trans. Faraday Soc. 57, 1603-1611 (1961).

37. Parsons, R. The kinetics of electrode reactions and the electrode material. Surf. Sci. 2, 418-435 (1964)

38. Santos E. \& Schmickler W. Catalysis in electrochemistry: from fundamental aspects to strategies for fuel cell development. (John Wiley \& Sons, 2011).

39. Walter, M. G. et al. Solar water splitting cells. Chem. Rev. 110, 6446-6473 (2010).

40. Wang, Q. et al. Scalable solid-state synthesis of highly dispersed uncapped metal (rh, ru, ir) nanoparticles for efficient hydrogen evolution. Adv. Energy Mater. 8, 1801698 (2018).

41. Pan, Y. et al. Cobalt nickel phosphide nanoparticles decorated carbon nanotubes as advanced hybrid catalysts for hydrogen evolution. J. Mater. Chem. A 4, 14675-14686 (2016).

42. Jiang, B., Liao, F., Sun, Y., Cheng, Y. \& Shao, M. Pt nanocrystals on nitrogendoped graphene for the hydrogen evolution reaction using Si nanowires as a sacrificial template. Nanoscale 9, 10138-10144 (2017).

43. Naruto, M. \& Saito, S. Cationic mononuclear ruthenium carboxylates as catalyst prototypes for self-induced hydrogenation of carboxylic acids. Nat. Commun. 6, 8140 (2015).

44. Zhang, L. et al. Atomic layer deposited Pt-Ru dual-metal dimers and identifying their active sites for hydrogen evolution reaction. Nat. Commun. 10, 4936 (2019)

45. Cabán-Acevedo, M. et al. Efficient hydrogen evolution catalysis using ternary pyrite-type cobalt phosphosulphide. Nat. Mater. 14, 1245-1251 (2015).

46. Gao, M.-R. et al. An efficient molybdenum disulfide/cobalt diselenide hybrid catalyst for electrochemical hydrogen generation. Nat. Commun. 6, 5982 (2015).

47. Hod, I. et al. A porous proton-relaying metal-organic framework material that accelerates electrochemical hydrogen evolution. Nat. Commun. 6, 8304 (2015).

48. Zheng, Y. et al. High Electrocatalytic hydrogen evolution activity of an anomalous ruthenium catalyst. J. Am. Chem. Soc. 138, 16174-16181 (2016).

49. Wang, J., Wei, Z., Mao, S., Li, H. \& Wang, Y. Highly uniform Ru nanoparticles over $\mathrm{N}$-doped carbon: $\mathrm{pH}$ and temperature-universal hydrogen release from water reduction. Energy Environ. Sci. 11, 800-806 (2018).

50. Li, K. et al. Enhanced electrocatalytic performance for the hydrogen evolution reaction through surface enrichment of platinum nanoclusters alloying with ruthenium in situ embedded in carbon. Energy Environ. Sci. 11, 1232-1239 (2018).

51. Rosca, I. D., Watari, F., Uo, M. \& Akasaka, T. Oxidation of multiwalled carbon nanotubes by nitric acid. Carbon 43, 3124-3131 (2005).

52. Tchoul, M. N., Ford, W. T., Lolli, G., Resasco, D. E. \& Arepalli, S. Effect of mild nitric acid oxidation on dispersability, size, and structure of single-walled carbon nanotubes. Chem. Mater. 19, 5765-5772 (2007).

53. Andrade, N. F. et al. Temperature effects on the nitric acid oxidation of industrial grade multiwalled carbon nanotubes. J. Nanopart. Res. 15, 1761 (2013).

54. Gheorghiu, C. C. et al. Chiral rhodium complexes covalently anchored on carbon nanotubes for enantioselective hydrogenation. Dalton Trans. 43, 7455-7463 (2014). 
55. Kresse, G. \& Furthmüller, J. Efficient iterative schemes for ab initio total-energy calculations using a plane-wave basis set. Phys. Rev. B 54, 11169-11186 (1996).

56. Kresse, G. \& Furthmüller, J. Efficiency of ab-initio total energy calculations for metals and semiconductors using a plane-wave basis set. Comput. Mater. Sci. 6, 15-50 (1996).

57. Kohn, W. \& Sham, L. J. Self-consistent equations including exchange and correlation effects. Phys. Rev. 140, A1133-A1138 (1965).

58. Perdew, J. P., Burke, K. \& Ernzerhof, M. Generalized gradient approximation made simple. Phys. Rev. Lett. 77, 3865-3868 (1996).

\section{Acknowledgements}

This research was supported by the Creative Research Initiative (CRI, 2014R1A2069102), BK21 Plus (10Z20130011057), Science Research Center (SRC, 2016R1A5A1009405) and Young Researcher (2019R1C1C1006650) Programs through the National Research Foundation (NRF) of Korea, funded by the Ministry of Science, ICT, and Future Planning. M.S.O. and N.P. appreciate NRF for the support (2019R1A2C2089332). The EXAFS experiments were performed in the PAL beamline (6D C\&S UNIST-PAL).

\section{Author contributions}

J.-B.B, J.M., and D.H.K. conceived and designed the project. D.H.K., J.M., J.-P.J., and H.J.N carried out the synthesis and characterizations. S.-J,K and D.H.K were involved in the EXAFS characterizations. D.H.K performed electrochemical analyses. M.S.O. and N.P. carried out the DFT study. D.H.K, J.M., and J.-B.B. wrote the manuscript and all authors discussed the results and commented on the paper.

\section{Competing interests}

The authors declare no competing interests.

\section{Additional information}

Supplementary information is available for this paper at https://doi.org/10.1038/s41467020-15069-3.

Correspondence and requests for materials should be addressed to J.M. or J.-B.B.

Peer review information Nature Communications thanks Meng Ni and other, anonymous, reviewers for their contributions to the peer review of this work. Peer review reports are available.

Reprints and permission information is available at http://www.nature.com/reprints

Publisher's note Springer Nature remains neutral with regard to jurisdictional claims in published maps and institutional affiliations.

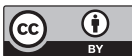

Open Access This article is licensed under a Creative Commons Attribution 4.0 International License, which permits use, sharing, adaptation, distribution and reproduction in any medium or format, as long as you give appropriate credit to the original author(s) and the source, provide a link to the Creative Commons license, and indicate if changes were made. The images or other third party material in this article are included in the article's Creative Commons license, unless indicated otherwise in a credit line to the material. If material is not included in the article's Creative Commons license and your intended use is not permitted by statutory regulation or exceeds the permitted use, you will need to obtain permission directly from the copyright holder. To view a copy of this license, visit http://creativecommons.org/ licenses/by/4.0/.

(C) The Author(s) 2020 